\title{
WIDE-BAND CURRENT TRANSDUCERS USING COMMON MODE FILTER CHOKES
}

\author{
Alex Van den Bossche ${ }^{1}$, Mohannad Jabbar Mnati ${ }^{2}$ \\ ${ }^{1}$ Electrical Engineering Laboratory, Ghent University, Belgium \\ ${ }^{2}$ Department of Electronic Technology, Institute of Technology Baghdad, Middle Technical University, Iraq \\ ${ }^{3}$ Flanders Make, the Strategic Research Centre for the Manufacturing Industry, Lommel, Belgium
}

\section{Introduction}

Wide band target:

- Low frequency: high flux, high $\mu$ r

- High frequency: propagation in the winding

- Nanocrystalline material

- By chance possible to use common mode chokes

\section{Electrical properties given by}

the manufacturer

\begin{tabular}{|c|c|c|c|c|c|}
\hline Properties & Test conditions & & Value & Unit & Tol. \\
\hline Inductance & $10 \mathrm{kHz} / 0.1 \mathrm{~mA}$ & $\mathrm{~L}$ & $2 \times 3.0$ & $\mathrm{mH}$ & $+50 \% /-30 \%$ \\
\hline Rated current & $@ 70^{\circ} \mathrm{C}$ & $\mathrm{I}_{\mathrm{R}}$ & 26.0 & $\mathrm{~A}$ & $\max$. \\
\hline DC Resistance & $@ 20^{\circ} \mathrm{C}$ & $\mathrm{R}_{\mathrm{DC}}$ & $2 \times 4.5$ & $\mathrm{~m} \Omega$ & $\max$. \\
\hline Rated voltage & $50 \mathrm{~Hz}$ & $\mathrm{U}_{\mathrm{R}}$ & 250 & $\mathrm{~V}(\mathrm{AC})$ & $\max$. \\
\hline Insulation test voltage & $50 \mathrm{~Hz} / 5 \mathrm{~mA} / 2 \mathrm{sec}$. & $\mathrm{U}_{\mathrm{T}}$ & 1500 & $\mathrm{~V}(\mathrm{AC})$ & \\
\hline
\end{tabular}

\section{Common mode choke}

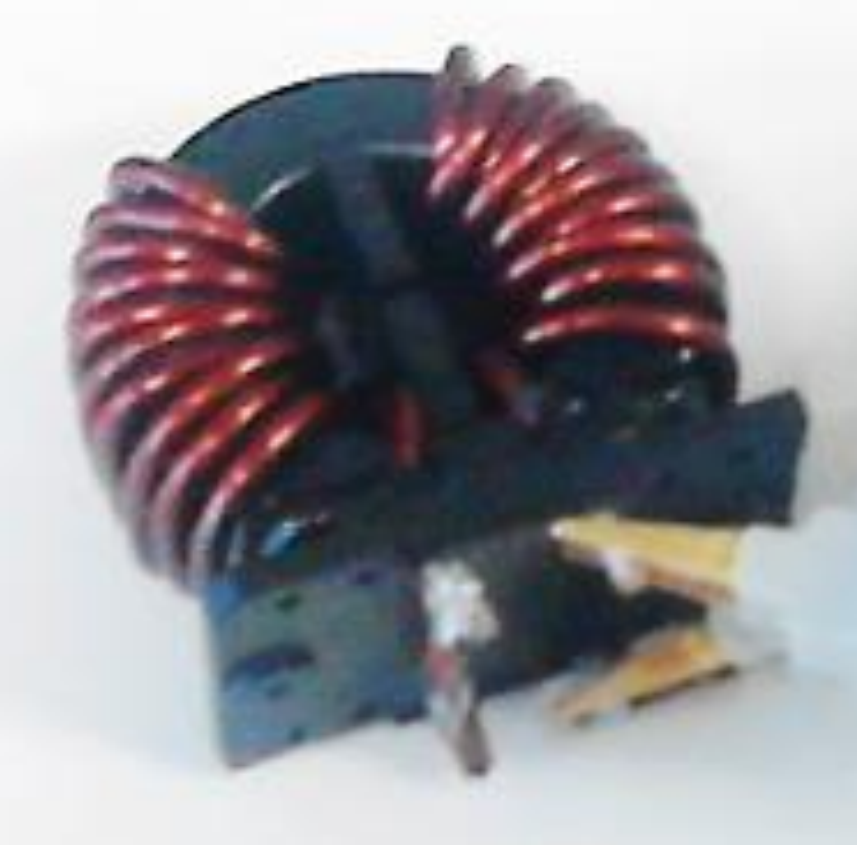

Principle scheme

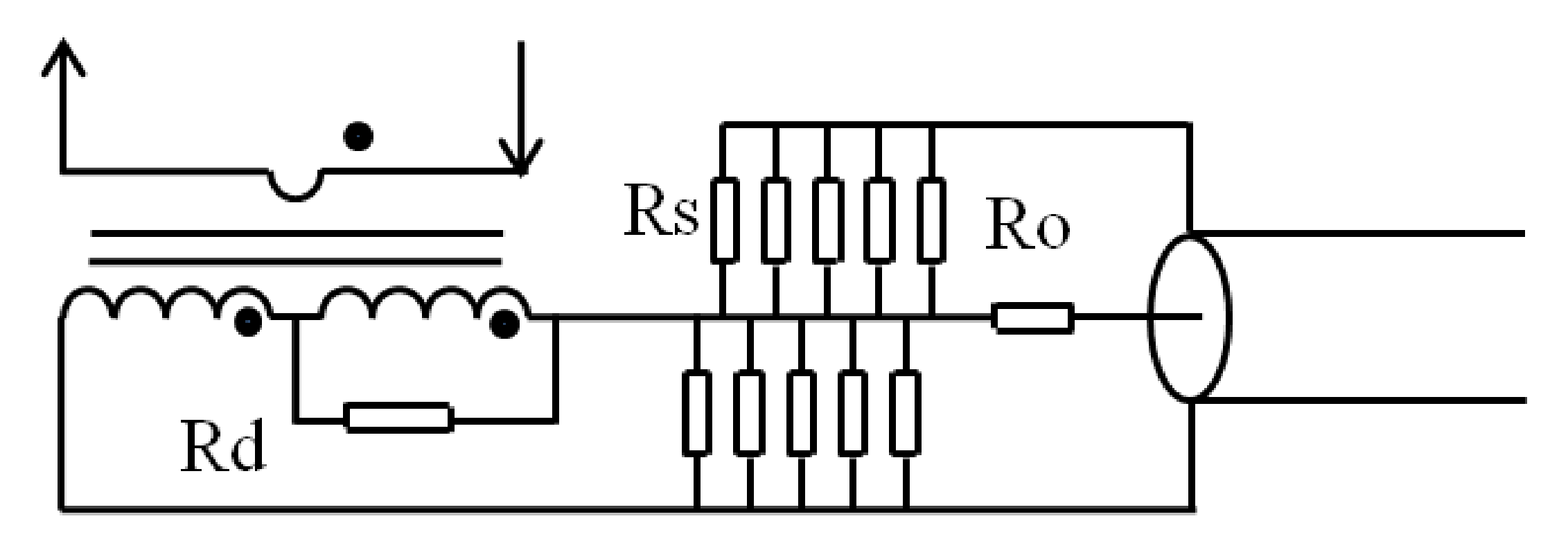

2x 22 turns, $180 \Omega 10 \times 0.22 \Omega 220 \Omega$

Model for the resonance of leakage inductance

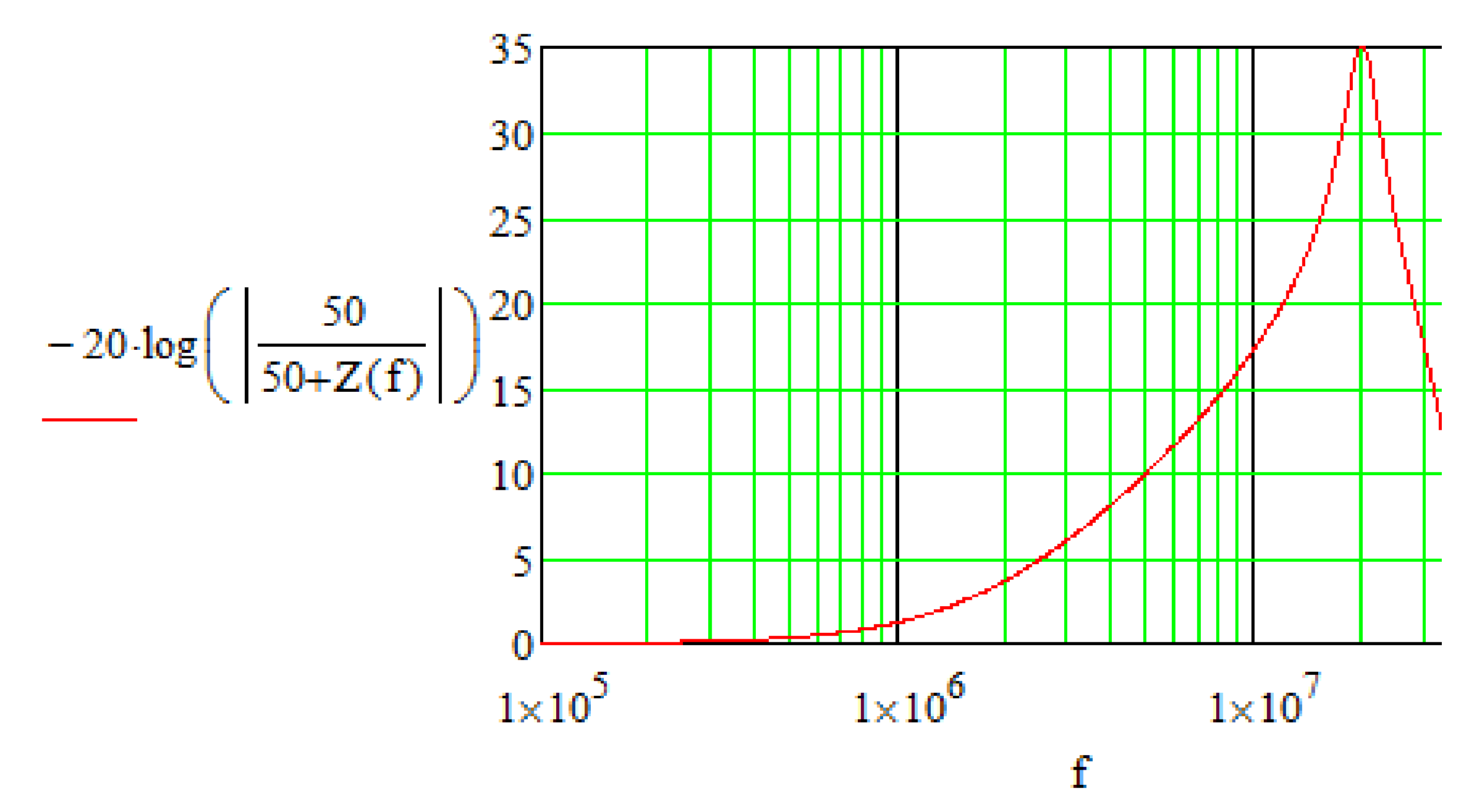

PCB Lay -out

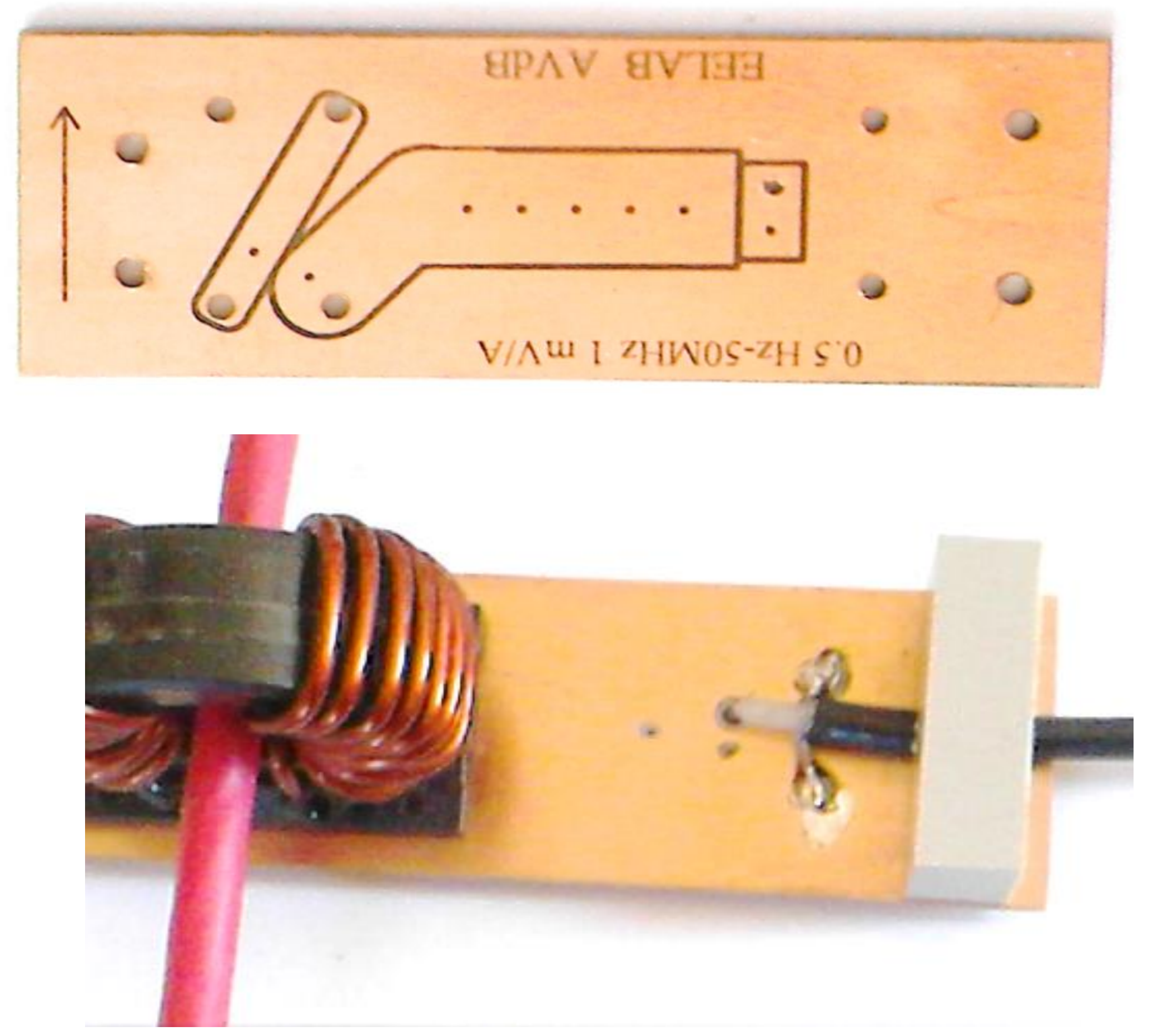

Manufacturer data of insertion loss with a $50 \mathrm{ohm}$ load

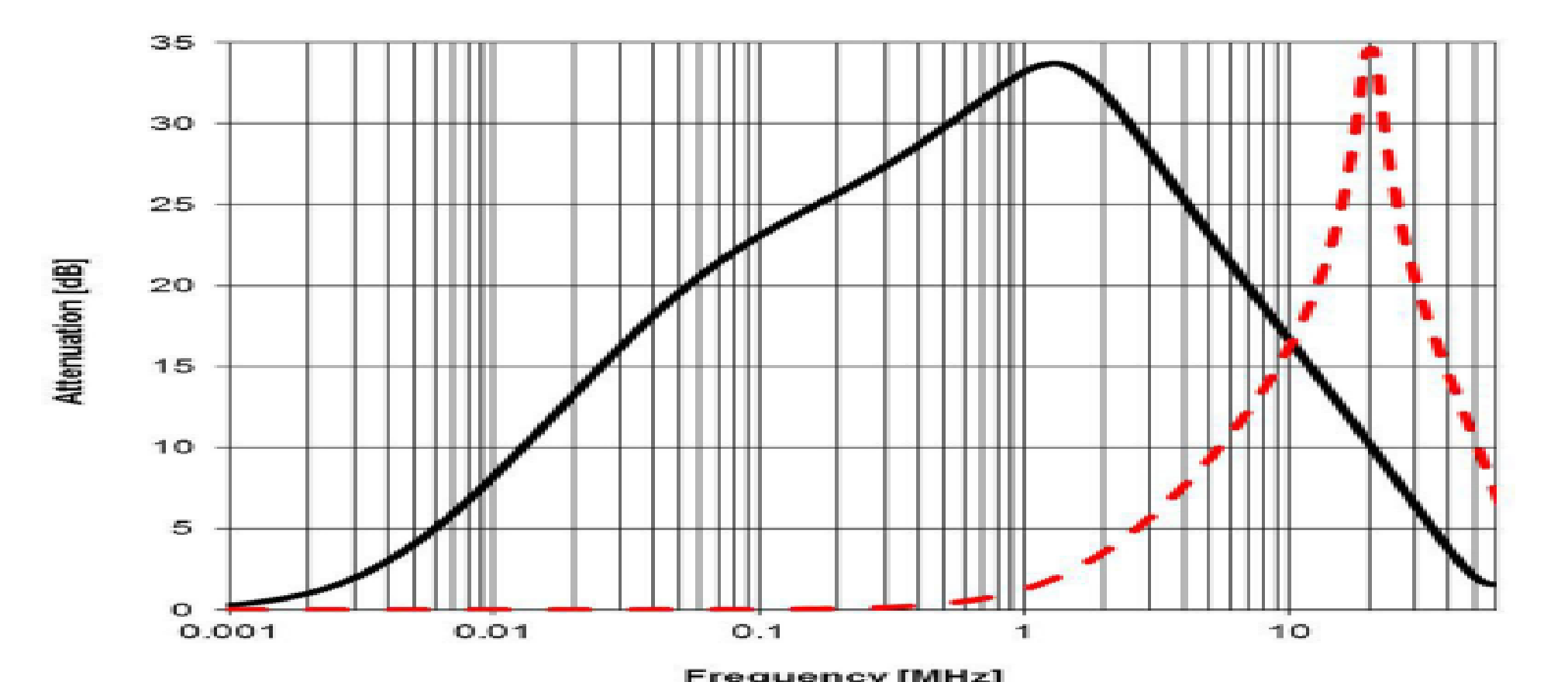

Operation limits

Saturation, shunt temperature, eddy current

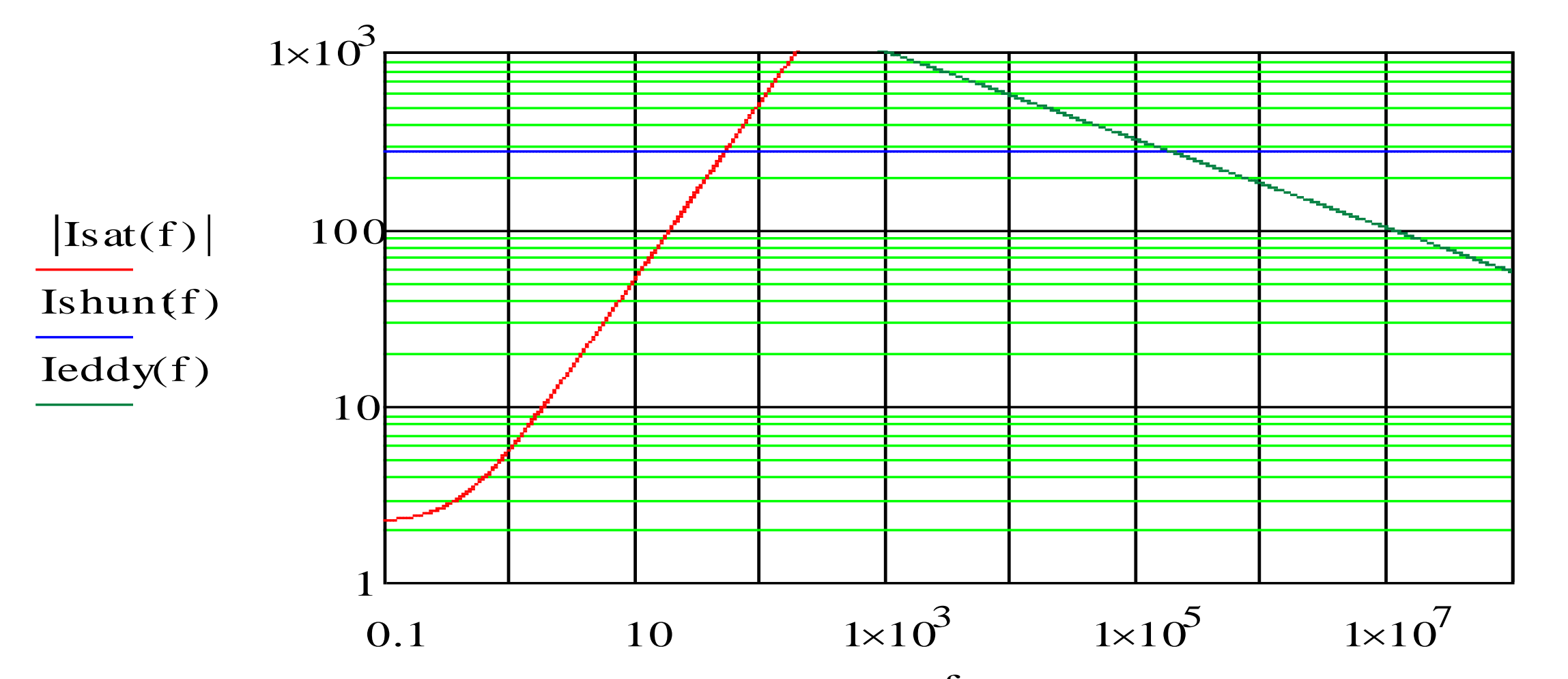

TF using the transmission line model

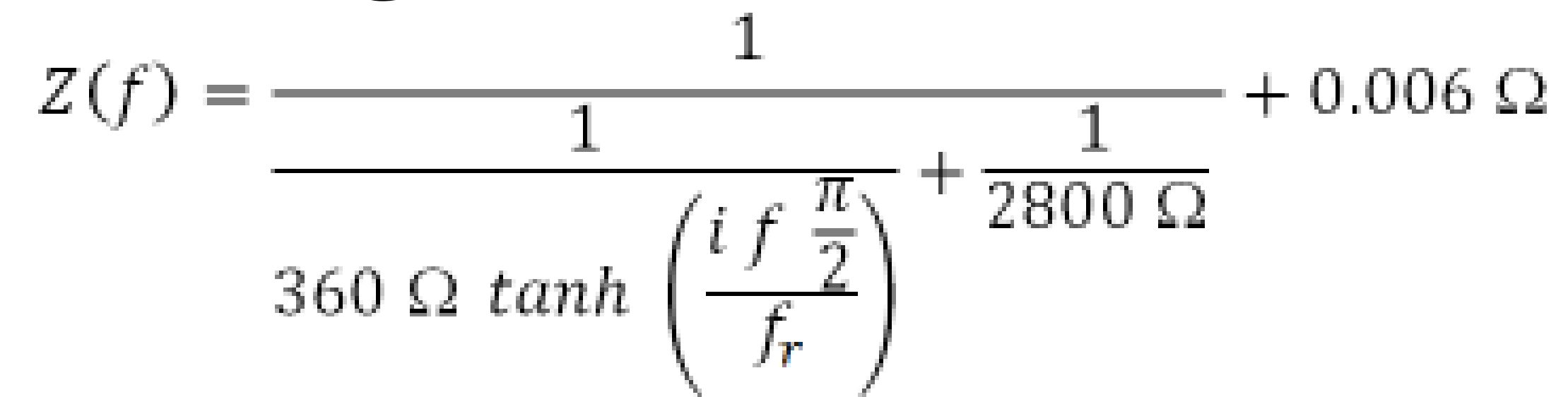

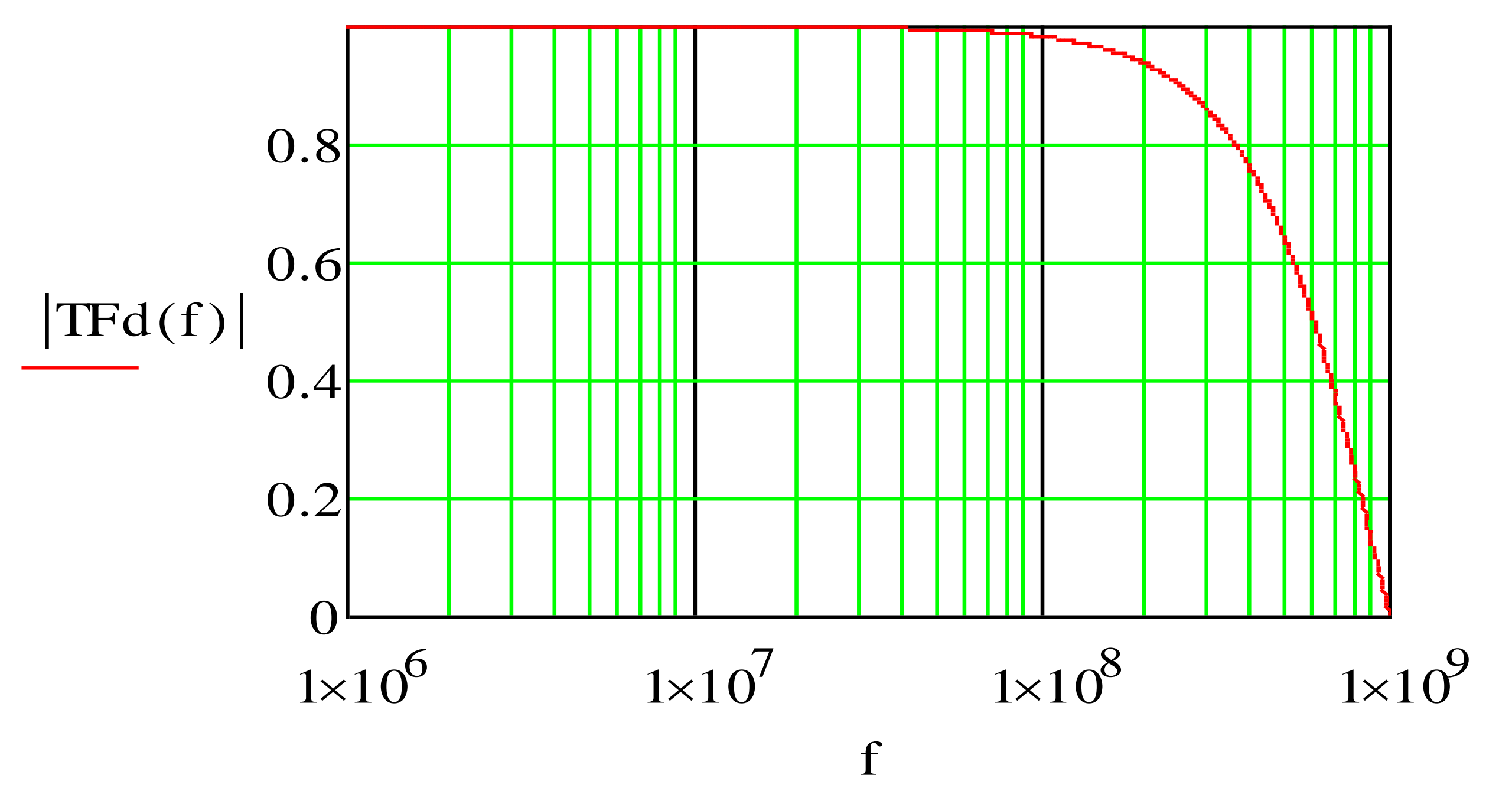

Transfer using 10 times higher shunt resistance

Real design goes to lower freq.

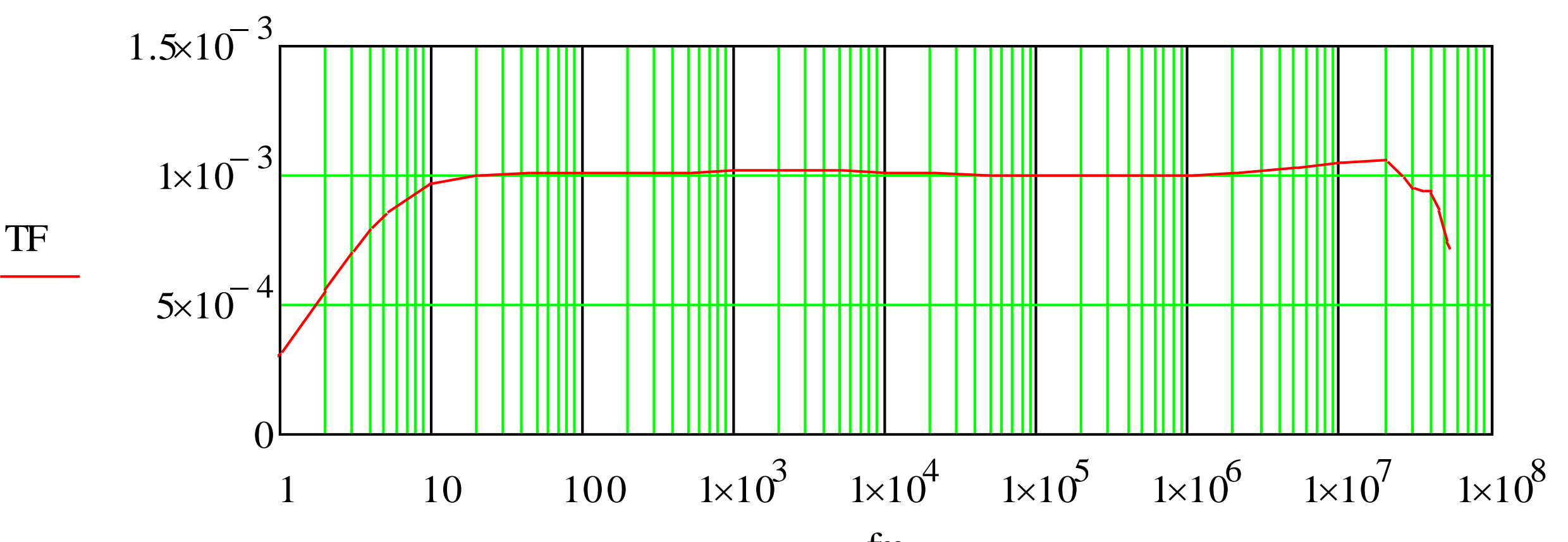

\section{Conclusions}

Strong points:

$>$ Wide band: from $0,5 \mathrm{~Hz}$ up to $50 \mathrm{MHz}$ is possible, ratio $\mathbf{1 0}^{\wedge} \mathbf{8}$;

$>$ Low phase shift in a wide frequency range.

$>$ Known limits of operation.

$>$ High current capability

$>$ Single pulse operation is possible

Weak points::

$>$ No DC output, saturation at 2.2A DC at input;

$>$ Saturates early at low frequency

$>$ Low output voltage level 